\title{
Hacia una antropología sistémica desde los Andes
}

Artículos originales: ANTROPOLOGÍA

Recibido: 28/08/2020

Aprobado: $13 / 10 / 2020$

Publicado: 05/02/2021
Luis Alberto Suárez Rojas

Universidad Nacional Mayor de San Marcos Isuarezr@unmsm.edu.pe

\begin{abstract}
RESUMEN
Proponemos revisar lo global como un sistema complejo, tenso y dinámico, configurado tanto por nuevos actores (bancos, corporaciones globales) como por arquitecturas institucionales, geografías de poder y redes transnacionales. En suma, sugerimos una aproximación crítica de la globalización. En términos de hipótesis, sostenemos que una antropología sistémica se apoya en la lógica del sistema-mundo, tomando en consideración el pensamiento latinoamericano y la teoría critica transformativa. Asumimos que lo global ni es abstracto ni es una marea que arrastra con formas locales. Lo global es una experiencia concreta, a partir del cual emergen múltiples respuestas y fricciones.
\end{abstract}

Palabras clave: Globalización; capitalismo; enfoque sistémico; teoría antropológica; etnografía multisituada; teoría social.

\section{Possibilities of Systemic Anthropology from the Andes}

\begin{abstract}
We propose to review the global as a complex, tense and dynamic system, configured both by new actors (banks, global corporations) and by institutional architectures, geographies of power and transnational networks. In short, we suggest a critical approach to globalization. In terms of hypotheses, we argue that a systemic anthropology is based on the logic of the world-system, taking Latin American thought and critical transformative theory into consideration. We assume that the global is neither abstract nor a tide that drags with local forms. The global is a concrete experience, from which multiple responses and frictions emerge.
\end{abstract}

KeYwords: Globalization; capitalism; systemic approach; anthropological theory; multisite ethnography; social theory. 


\section{Introducción}

E l presente artículo de investigación está basado en un trabajo más amplio y de largo aliento. Mi interés central es compartir con los lectores las ideas centrales de mi tesis doctoral titulada "Antropología sistémica. Aproximaciones críticas de lo global/local con una perspectiva poscolonial desde los Andes» (Suárez, 2018). En este trabajo estoy convencido que como sociedad y como generación nos toca vivir los rápidos avances tecnológicos que se vienen produciendo en muchos ámbitos de la vida social, material y ambiental. En un tiempo no muy lejano ya no causará asombro la lectura de algunos escritores como Isaac Asimov, Robert A. Heinlein o Arthur C. Clarke. El desarrollo de nuevas dinámicas tecnológicas ha provocado que filósofos y sociólogos como Michel Callon, Bruno Latour y otros, reivindiquen el análisis de la relación entre los humanos y los objetos (máquinas y otros dispositivos). Así en el 2005, Latour publicó su texto "Reassembling the social: An introduction to actor-network-theory" (2005) en el cual sugiere redefinir lo «social» como el resultado de interacciones concretas y materiales entre elementos que son en sí no-sociales, a partir de la mediación tecnológica. Este ejercicio busca comprender la relación entre la sociedad, la tecnología y la ciencia a fin de conocer las implicancias de los procesos de hipermediación tecnológica en la sociedad y la cultura. (Latour, 2005), (Feenberg \& Callon, 2010).

Es evidente que las ciencias sociales no pueden estar de espalda a esta fuerza imparable de cambios materiales, tecnológicos y científicos, y menos aún la antropología que nos permitiría examinar, no solo los aspectos simbólicos, relacionales y significados, también las profundas asimetrías que supone la relación entre la mediación tecnológica, la ciencia y el poder, desde una perspectiva histórica y comparada. Un ejemplo de los dilemas que plantea la tecnología y la digitalización a la sociedad contemporánea en democracia, fue el escándalo provocado por la empresa Facebook y la Cambridge Analytica (CA). Esta empresa utilizaba la minería de datos («Knowledge Discovery in Databases» o KDD) con la comunicación estratégica. Cambridge Analytica utilizó los datos personales (no autorizados) de los usuarios para deslizar una campaña a favor de Trump. Esto provocó que Mark Zuckerberg fuera interrogado por el congreso norteamericano y la empresa Cambridge Analytica cerrara. Así, sociedad, política y tecnología están cada vez más entrelazados, a veces de manera beneficiosa y otras peligrosa.

A comienzo de la década de 1990, el notable antropólogo francés Marc Augé publicó su largo ensayo titulado "Non-lieux. Introduction à une anthropologie de la surmodernité» (1992). Aquí destacó la idea de que el mundo (en su espacialidad) se encoge y el tiempo se acelera, asimismo emergen lugares para el anonimato desligados de la historia y la identidad, y donde se divisa cada vez más el lujo, la extrema riqueza y la extrema pobreza como mundos que coexisten en medio de las lógicas de reproducción de la precariedad del empleo y se exaltación de la individualidad y la soledad. Esa es la sobremodernidad (Augé, 1992), diagnóstico alternativo a la globalización y la mundialización como prefieren referirse los franceses. Paralelamente, desde el rincón de la filosofía alemana Peter Sloterdijk planteó una serie de provocadoras reflexiones sobre las esferas y las espumas. Desde su perspectiva la sociedad occidental se caracteriza por coexistencias individuales como «las burbujas en la espuma, es decir, las parejas y hogares, los equipos y comunidades de supervivencia son microcontinentes constituidos autorreferencialmente» (Sloterdijk, 2014) con formas de díadas y configuraciones espacio-anímicas complejas. A este grupo se suman otros intelectuales como Ulrich Beck, Zygmunt Bauman, Jean Baudrillard, Slavoj Žižek, entre otros.

\section{Avances tecnológicos y desigualdades sociales.}

Han pasado más de 25 años del diagnóstico de la sobremodernidad. Hoy estamos viviendo la era del bitcoin y de las criptomonedas, la búsqueda de exoplanetas, el lanzamiento de sondas espaciales, el advenimiento de la inteligencia artificial (IA) y de la expansión de nuevas formas de relación social mediadas por la tecnología. En este campo de innovaciones destaca el desarrollo acelerado de la biotecnología, la nano tecnología, la revolución de las telecomunicaciones y la digitalización de la sociedad. Muchos dirían que tenemos «avances» pero también registramos retrocesos en muchos campos, y ahí está la paradoja. Hoy vivimos un tiempo donde el universo se compara con un holograma, así lo holográfico y el multiver- 
so ganan terreno, dejando atrás la distinción entre lo analógico y lo digital.

Desde hace algunos años Raymond Kurzweil es considerado el heredero de Thomas Edison. Ha compartido sus ideas futuristas, realizando más de 147 predicciones sobre la introducción de nueva tecnología con un acierto de más del $80 \%$. Antes de comenzar el siglo veintiuno predijo que para la siguiente década se utilizaría coches de manera autómata; ciertamente, la empresa Uber, valoriza en 70, $000 \mathrm{mi}$ llones de dólares, lanzó de manera experimental en la ciudad de Pittsburgh una pequeńa flota de carros autómatas. Al mismo tiempo que otras empresas siguen explorando la posibilidad de los autos autómatas como por ejemplo la empresa Tesla.

Raymond Kurzweil publicó un sugerente libro titulado "The Singularity is near. When humans transcend biology" (2010). En este trabajo pone al centro del debate el concepto de Singularidad. Para este futurólogo la vida humana logrará superar las limitaciones de la biología, en especial nuestro cerebro, de este modo venceremos al tirano del tiempo y será posible disfrutar de la longevidad. Para Kurzweil viviremos un tiempo donde las máquinas y los humanos lograran una mayor interacción. Además, se producirán cambios radicales en la manera cómo los humanos aprenderán, trabajarán o harán la guerra. Incluso asegura que para el 2030 los cerebros humanos podrán conectarse a la nube e interactuar con múltiples interfaces.

El futuro parece prometedor y aun cuando se producen los avances tecnológicos las brechas sociales se incrementan y con ello la polarización social. La Agencia de Naciones Unidas para los Refugiados (ACNUR) señaló que para el 2016, existía un total de 65,6 millones de personas debido a la guerra y la violación de derechos humanos. En el 2016, el secretario general de Naciones Unidas, Ban Ki-moon, en su visita a Lesbos criticó la construcción y cierre de fronteras ante los miles de solicitantes de asilo de países como Siria, Irak y Afganistán. En este escenario, no es difícil jugar con distopías, de hecho, la literatura y el cine vienen ofreciendo más narrativas de esta naturaleza. Hoy buscar otros mundos donde sea posible la reproducción de la vida humana es moralmente aceptada y pensada como una conquista más del hombre occidental. Así, la búsqueda de exoplanetas está siendo motivada por la búsqueda de otros mundos alternativos a la tierra, lo que genera un costo de 550 millones de dólares. En esta carrera están involucradas las agencias como la Nasa, la Agencia Espacial Europea y Rusia.

Occidente está muy orgulloso de los aportes de la ciencia y la tecnología al mundo. No falta quienes aseguren que toda la vida en el futuro estaría regida por la ciencia. Michio Kaku, uno de los físicos más reconocidos del mundo, catedrático de física teórica en el City College de New York, aseguró en 1996 que en el futuro tendríamos gafas conectadas a internet, vehículos sin conductor y pagos por el teléfono. Hoy todo eso ya es parte de nuestra cotidianidad. Michio Kaku asegura que para el futuro los chips serán muy baratos, los tendremos en la ropa, en el cuerpo, usaremos papel electrónico para pagar, tendremos ordenadores desechables, etc. (Kaku, 2015), (Kaku, 2016).

Sin embargo, muy a pesar del orgullo de occidente basado en la ciencia. La realidad social evidencia profundas desigualdades en el resto del mundo. En el presente, registramos el avance galopante de la biotecnología, las tecnologías digitales y las criptomonedas, al tiempo que vivimos con crudeza los efectos de una economía al servicio del $1 \%$. Así, no solo vivimos la pobreza extrema, también la riqueza extrema. En el 2016, OXFAM circuló un informe titulado «una economía al servicio del 1\%», el cual señala que para el 2010, alrededor de 388 personas poseían una riqueza equivalente a la riqueza de 3,600 millones personas juntas, es decir más de la mitad de la población más pobre en el planeta. En cinco años más tarde la cifra de las personas más ricas del mundo se redujo, así pasamos de 388 a 62 personas, lo que significa cerca de 1,76 billones de dólares. El reporte sugiere que «desde el inicio del presente siglo, la mitad más pobre de la población mundial sólo ha recibido el $1 \%$ del incremento total de la riqueza mundial, mientras que el $50 \%$ de esa nueva riqueza ha ido a parar a los bolsillos del 1\% más rico» (OXFAM, 2016). Por su parte, el economista francés Thomas Piketty sugiere que esta es una primera regularidad, puesto que la «distribución de la propiedad del capital y de los ingresos resultantes es sistemáticamente mucho más concentrada que la de los ingresos del trabajo» (Piketty, 2014, pág. 267). Para este economista, aunque la lista de Forbes no siempre es una lista confiable sobre la súper riqueza, señala que esta clasificación estuvo dominada por japoneses entre 1987 y 1995 , 
luego hasta el 2009 por un estadounidense y luego al presente por un mexicano. Además, subraya que en 1987 había solo cinco multimillonarios por cada 100 millones de habitantes y para el 2013 había un total de 30 multimillonarios (Piketty, 2014, pág. 676). En un espacio de 26 años (1987 al 2013) los multimillonarios pasaron de tener control de $0,4 \%$ de la riqueza privada mundial al 1,5\%; así mismo, el $1 \%$ de los ricos posee un patrimonio promedio de 3 millones de euros (Piketty, 2014, pág. Ibídem). Las profundas desigualdades no solo están en relación a las categorías culturales y simbólicas, sino también a la capacidad que poseen los grupos para monopolizar los recursos (Tilly, 2000), en ese sentido, enfatiza el papel de las herencias. La fortuna no solo está en relación al mérito, sino al patrimonio heredado «a menudo, los patrimonios heredados logran obtener un rendimiento muy elevado por el simple hecho de su cuantía inicial» (Piketty, 2014, pág. 485), en el fondo lo que hay que examinar es cómo estas fortunas se multiplican y se perpetúan «más allá de todo límite y de toda posible justificación racional en términos de utilidad social» (Piketty, 2014, pág. 488). En muchos casos es difícil de rastrear debido usualmente el dinero pasa a los espacios extraterritoriales del capitalismo conocido como los «paraísos fiscales».

En el 2016, se produjo uno de los mayores escándalos en las finanzas llamado "Panama Papers». Esto constituyó la mayor filtración de documentos de la firma de abogados panameña Mossack Fonseca. Esto significó la entrega de 2,6 terabytes de información al periódico alemán Süddeutsche Zeitung. Se han identificado alrededor 13.4 millones de documentos de la firma de abogados Bermudan Appleby, compañía con 119 años de vida al servicio de las grandes corporaciones del mundo. Esta masiva filtración permitió identificar que Yuri Milner, multimillonario ruso invirtió en Facebook y Twitter, pero los bancos que financiaban a éste, estaban controlados por el Kremlin, vínculos que no fueron claros pues los rastros se perdían en las compañías ficticias extraterritoriales. Incluso, Tim Cook presidente ejecutivo de Apple, acumuló más de $\$ 128$ mil millones en ganancias no gravadas en alta mar. En el 2014, este ejecutivo declaró ante el senado de EEUU por las ganancias se enmascararon en empresas fantasmas irlandesas. También reveló que incluso la reina Isabel II realizó inversiones en fondos extraterritoriales (casi $13 \mathrm{mi}$ - llones de dólares). Asimismo, Stephen Bronfman, asesor del primer ministro de Canadá Justin Trudeau y principal recaudador de fondos (heredero de la fortuna de Seagram) escondió millones de dólares en las Islas Caimán e Israel (Richardson, 2017). En este contexto, Bernie Sanders, senador estadounidense de Vermont, ha señalado que el escándalo financiero y político de "Panama Papers», está revelando cómo una «oligarquía internacional» compuesta por grupo reducido de multimillonarios está enriqueciéndose a costa de los impuestos en sus países. Según Sanders el aspecto más crítico es el ascenso vertiginoso de la oligarquía internacional. Así, este escándalo es la muestra del papel deplorable de los multimillonarios quienes evaden los impuestos y ocultan las verdaderas ganancias (Pilkington \& Smith, 2017). El reciente trabajo de Naomi Klein recoge los datos proporcionados por James Henry, miembro de la organización Tax Justice Network, quien asegura que para el 2015 la riqueza individual en los paraísos fiscales repartidos en todo el globo era entre 24 y 36 billones de dólares (Klein, 2017, pág. 47). David Harvey también ha encendido las alarmas sobre una oligarquía global (Harvey, 2016).

El caso de Panama Paper revela la otra cara del neoliberalismo. La otra cara de este modelo hegemónico lo constituyen esos tratos bajo la mesa y el coqueteo constante con los llamados «mundos extraterritoriales» que no son más que espacios localizados por fuera de la regulación y que facilita ocultar parte de la extrema riqueza. Como ya habíamos sugerido antes (Suárez, 2018) podemos decir, siguiendo a John Urry, que estos son zonas económicas especiales que revelan los límites de la democracia y que en el fondo constituyen parte del ensemble de flujos, jurisdicciones secretas, mercados financieros especulativos. Territorios liberados que coexisten con el lujo extremo de los ricos y famosos. Todo esto no es sino el rasgo más certero del neoliberalismo, ahí encontramos una constante deslocalización y un régimen secretista (Urry, 2017), (Suárez, 2018).

No solo John Urry ha estudiado ese mundo deslocalizado "offshore», también lo ha hecho Ronen Palan, profesor de economía política internacional en la Universidad de Birmingham, quien en el 2006 publicó su libro "The offshore world: sovereign markets, virtual places, and nomad millionaires.», aquí describe cómo se configura la economía extraterritorial, y 
explora hasta dónde lugares como las Islas Caimán o Zúrich constituyen un centro bancario «extraterritorial». Aquí se produce un juego peligroso entre lo regulado y lo no-regulado. Su propuesta sugiere que la economía extraterritorial produce un mercado nuevo y soberano, que en ultimo término debilita al Estado-nación y fortalece el nomadismo del capital (Palan, 2006).

Ante este escenario de nuevos ricos, acumulación y riqueza extrema, una serie de movimiento convergen en lo que se llamó "Occupy Wall Street», este movimiento derivó en parte del Movimiento 15-O, Global Revolution, y que para el 15 de octubre del 2011 logró involucrar a 1051 ciudades de 90 países en el marco de las protestas ¡Democracia real ya! Así se registraron una serie de protestas en España, Bélgica, Francia, Italia, Chile, México y otros países del globo. En este clima de protestas y rebeldía surgió el movimiento «Occupy Wall Street» en el 2011, enarbolando la frase: "We are the 99\%». El movimiento Occupy Wall Street fue cubierto por muchos medios. Cientos de activistas se congregaron en las escalinatas del Federal Hall de Nueva York, frente al edificio de la Bolsa de Valores. Paul Krugman, eminente profesor de Economía en Princeton y premio Nobel 2008, sugirió que el 0,1\% de quienes detentan la súper riqueza del mundo son mandamases de empresas y embaucadores financieros. Según un análisis reciente, el $43 \%$ de la superélite son ejecutivos de empresas no financieras; el $18 \%$ se dedica a las finanzas, y otro $12 \%$ son abogados o están en el sector inmobiliario (Krugman, 2011). Un año después, Volscho y Nathan (2012) analizaron los factores que impulsaron el posicionamiento y crecimiento de una clase súper rica en EEUU. Este equipo de investigadores aseguró que el aumento de los súper ricos es el resultado de cambios a la derecha en el Congreso de EEUU, el declive de los sindicatos, tasas impositivas más bajas en los altos ingresos, mayor apertura comercial y burbujas de activos en los mercados de valores e inmobiliarios (Volscho \& Nathan, 2012).

Pero como señalan nuestros maestros de la sospecha, el devenir del capitalismo se somete a un ritmo cíclico. El devenir de una elite, de una oligarquía mundial y súper-ricos no es ningún fenómeno nuevo. Thorstein Veblen (1899) había desarrollo una aproximación crítica desde la economía y la moral a lo que él denominó la «clase ociosa» donde se conju- ga la riqueza, el hedonismo y la prepotencia. Por otro lado, Napoleoni nos recuerda que ese mundo de los nuevos ricos, de la clase ociosa como decía Veblen, son actualmente los oligarcas rusos, los futbolistas europeos, los magnates chinos e indios, los actores y las estrellas del pop, la televisión y los financieros, estos últimos han logrado adueńarse de una cantidad exorbitante de dinero (Napoleoni, 2008).Todo esto no es sino manifestación de lo que ha venido a llamar «economía canalla».

Desde este enfoque, el mercado global es el «caldo de cultivo» de la economía canalla. Una expresión de ello, por ejemplo, es el oro que proviene de la minería ilegal y termina en manos de la banca suiza, o cuando las tierras raras extraídas de minas informales del Congo terminan en las Tablet y en los teléfonos celulares inteligentes en el mundo. La economía canalla es producto de una transformación donde la política y la economía se separan, dicho de otro modo, la política pierde el control de la economía así «el mercado había roto las cadenas de la política (...) la economía se había convertido en una fuerza salvaje» (Napoleoni, 2008, pág. 31), es —al decir de Napoleoni- un retorno a una economía salvaje, en medio del desmantelamiento del comunismo y el surgimiento de la globalización (Napoleoni, 2008). La galopante desigualdad de los ingresos es una expresión de esa economía canalla, pero sobre todo esta es el lado oscuro de un nuevo orden mundial.

\section{Lo global como objeto antropológico}

Desde mi perspectiva, hay una visión muy hegemónica de la globalización. Algunos de plano satanizan todo lo que venga de la globalización y otros por el contrario alaban los beneficios como el flujo rápido y casi instantáneo de capitales y riqueza. Para los grandes empresarios la globalización tiene un carácter moral y ético, representa la victoria civilizatoria y conquista del espacio y el tiempo. La globalización constituye una narrativa y un discurso poderoso de quienes están de lado del poder y la hegemonía. Para Sloterdijk la globalización puede entenderse como fenómeno social de larga data, pero como evento político debe ser revisado a partir de nuevas dinámicas como el neoliberalismo y la economía de mercado (Sloterdijk, 2014) (Suárez, 2018). Por esa razón, para 
Wallerstein la globalización no es algo nuevo o apartado del capitalismo, es una fase más de la expansión capitalista (Suárez, 2018).

Pero, ¿acaso la globalización en tanto moda académica debería desestimar las contribuciones de perspectivas como el sistema-mundo y los aportes de Braudel o Wallerstein?, ¿globalización puede entenderse desde su complejidad sistémica? Desde luego apuesto por recuperar los aportes de Braudel (1980) y Wallerstein (2002), ambos son útiles aún para entender que el mundo moderno puede ser pensado desde la lógica de un sistema-mundo capitalista, iluminando compleja interacción entre centros y periferias. Solo que es necesario hacer algunos virajes para ir más allá de las limitaciones del modelo y lograr cierta revitalización de un marco analítico que permita repensar lo global como objeto antropológico en el marco de la expansión del capitalismo (Suárez, 2018).

Al respecto de lo global, muchos análisis se inclinan por pensar la determinación de lo global sobre lo local. Sin embargo, este proceso no es unidireccional. Así, antropólogos como Marshal Shalins (1988) analizaron la relación dialéctica entre el sistema-mundo moderno y las sociedades tribales de Hawaii; esto supone tener en consideración los actores coloniales y las dinámicas de comercio transnacional que incluyen a China. Su trabajo de análisis ofrece la posibilidad de analizar cómo las poblaciones locales interactúan históricamente en el sistema-mundo, reelaborando sus repertorios culturales y los aspectos de la vida social. Del mismo modo, los trabajos de June Nash (1981, 1994, 2006, 2008) van más allá de las fronteras del «presente etnográfico», ubicando su ejercicio en el marco de la «larga duración» y en la lógica del sistema-mundo. De este ejercicio se desprende la idea que «lo local» se configura gracias a la dialéctica entre el pasado, el presente y los complejos campos de fuerzas y tensiones. Esta brillante antropóloga, reivindica el trabajo de campo, subrayando la importancia de encontrar formas de aterrizar el andamiaje conceptual de sistema-mundo, especialmente a partir de un abordaje etnográfico en las zonas periféricas. Esto supone enfrentar la complejidad cultural y sistémica de los bordes del sistema mundo, sus contradicciones y los sistemas de fuerza que envuelven al sistema mundo. Esto podría permitirnos una búsqueda analítica de las nuevas estrategias, reacomodos y tácticas de los actores en el sistema-mundo, los ejercicios de rebeldía y desorden anti-sistémico. Para Nash, el orden de integración global, no es un proceso armónico, sino cargado de fricciones y vaivenes, ponderando el papel de los movimientos sociales y sus luchas por construir una alternativa a la hegemonía neoliberal. Nash abre el camino para pensar los problemas locales desde una perspectiva global.

Por otro lado, Marcus (1995) tratar de conjugar una mirada histórica del proceso con el «aquí-ahora» (presente etnográfico) enfatizando la simultaneidad e interdependencia en procesos y las variables sociales y culturales que atraviesan lo local. En una perspectiva convergente, Ulf Hannerz (1987), aseguró que el «aquí y ahora» del etnógrafo debe considerar las interacciones culturales con procesos más macro, más globales, explorando la relación entre los flujos culturales transnacionales y la continua creatividad cultural en la periferia del sistema mundo. Hannerz (1987) abogó por una «macro-antropología de la cultura» que tuviera como marco el sistema-mundo, sin soslayar los sistemas de significado que no ocultan sus conexiones con los hechos de poder y la vida material. (Hannerz, 1987).

Por otro lado, Anthony D. King, en su trabajo "Culture, Globalization and the World System: Contemporary Conditions for the Representation of Identity» (1991), analizó la cultura y la globalización desde la perspectiva del sistema-mundo, entrelazado con otras perspectivas y categorías como raza, género, etnia, clase y nación. King reivindica las posibilidades del uso de la etnografía para comprender procesos macro. Aborda los flujos globales de capital y su interacción con los espacios locales como un proceso híbrido. Para King (1991) los procesos de producción cultural en el capitalismo no se dan de manera estable por lo que la globalización constituye una dinámica discontinua en el tiempo histórico. Desde otra perspectiva, el antropólogo hindú, Arjun Appadurai sugiere que lo local aparece como producto histórico pero determinado por los flujos globales que van de lo global a lo local. En realidad, lo que apreciamos es cómo lo local es producto de un proceso híbrido, irregular, de entrada y de salida, de re-significación y reelaboración por actores, que siempre son locales, pero están atravesado por fuerzas globales. Aquí hay que marcar una diferencia pues no creemos que la globalización constituya una marea que arrastra for- 
mando localidades, por el contrario, es un proceso más complejo de lo que creemos, de cierta forma es híbrido y altamente dinámico en términos culturales. En ese línea, antropólogos franceses como Cuillerai y Abélès (2002) sugieren que la «mondialisation» revela un conjunto muy heterogéneo de dispositivos culturales, ligados a la reapropiación de la modernidad, por lo cual, la globalización tendría efectos en términos geo-político y en el imaginario.

Otros investigadores como Robertson (1992) buscan ir más allá de la aparente antinomia: global/ local, sugiriendo un neologismo llamado glocalización. Este es un giro de tuerca que busca comprender globalización a partir de «la interpenetración de la universalización de la particularización y la particularización del universalismo» (Robertson, 1992, p. 100). En ese camino, Robertson propone reconocer que existen procesos como la macro-localización que supone la apertura a los límites de la localidad y al mismo tiempo, procesos de micro-globalización que incluye procesos globales en los ámbitos locales. Esta táctica analítica, no hace sino enfatizar la idea de que lo local y lo global interactúan de manera compleja, híbrida, irregular, y dinámica. Esto sería parte de una dimensión fenomenológica ambivalente, ambigua de nuestra contemporaneidad. Siguiendo en parte los trazos de Robertson (1992), investigadores como Assayag (2005) insisten en repensar nociones como el retorno de lo local, la civilización mundial, sistema-mundo, enfatizando la multidimensionalidad de la globalización. En ese campo, la antropología puede ayudar a repensar el discurso dominante de la globalización y comprender cómo la universalización reinventa la «diferencia» (Assayag, 2005, p. 274), (Ramos Santana, 2007). Aunque es necesario ir más allá solo de la diversidad cultural y la diferencia cultural como foco para reflexionar más sobre los procesos comparativamente en el marco de la globalización neoliberal. Alabar la diferencia cultural podría soslayar problemas que se dan de manera simultanea en los bordes del sistema mundo, decir que se producen los mismos efectos en todas partes sería caer en una sociología espontánea, pero no abordarlos de manera compara para realmente conocer las implicancias de la maquina global sobre las experiencias locales, sería perder la oportunidad para el avance de las ciencias sociales (Suárez, 2018).
Desde otra perspectiva, Jonathan Friedman sugiere una antropología de lo global, considerando procesos y relaciones sistémicas globales. Su propuesta aborda la estructura de las condiciones de producción cultural, la profundidad histórica de la globalización, las relaciones globales y su capacidad formativa. El ejercicio de Friedman trata de construir una táctica analítica donde los procesos de larga duración son fundamentales, por ejemplo, en el caso de la identidad. En su razonamiento, durante la hegemonía de un núcleo civilizatorio la identidad es arrinconada y cuando se produce la declinación de tal hegemonía vuelve a producirse una reaparición de la identidad cultural. Intelectuales latinoamericanos también han logrado contribuciones significativas. Es imposible soslayar las contribuciones de Canclini y Gustavo Lins Ribeiro (2010) quienes enfatizan la relación entre la globalización y la economía política capitalista. La globalización desde abajo, supone retener categorías como el transnacionalismo, territorio y movimientos sociales.

Otro intelectual importante en este campo es Arturo Escobar a partir de su aproximación critica del lugar. En su mirada, si bien reconoce que hay un discurso dominante, asume que la globalización es una marea que arrasa con todo a su paso. Aunque su ejercicio analítico le permite poner en evidencia el papel de los movimientos sociales y los actores sociales quienes la política, la ecológica, la centralidad del lugar, la identidad, la historia, y otros modos de articularse con la naturaleza. Desde esta perspectiva, el lugar antropológico se revela atenazado por complejos flujos de mercancías e imaginarios que circulan, atravesados por reivindicaciones políticas que en fondo critican el núcleo de la hegemonía del poder. En esa línea, las contribuciones de Sassen (2007, 2010, 2015) son fundamentales para organizar un potente discurso crítico a la coexistencia de formas de desarrollo con dinámicas más dolorosas relacionadas a las expulsiones y las lógicas brutales del capitalismo que coexisten y se reproduce de manera tensa en los espacios locales.

La globalización es en el fondo deslocalización del capital, la industria, los servicios y las nuevas de pobreza y desigualdad, todo esto se experimenta en lo local. Por esa razón muchos de los estudiosos de la globalización, en la fase reciente, señalan que debemos tener cuidado de no caer en la trampa de la 
globalización como abstracta, cuando en realidad, en los espacios locales es concreta y se viven sus efectos como sus implicaciones directas. La globalización es un proceso concreto con implicancias locales, dicho de otra manera, es necesario reclamar la idea de entender las implicancias sociales y culturales de la experiencia local de la globalización.

\section{Elementos para una Antropología Sistémica}

De esta manera, mi propósito es construir una arquitectura teórica que me permita proponer una forma de abordar y pensar las implicancias socio-culturales y territoriales de lo global-local a través de una antropología sistémica, anclada en el pensamiento sistémico, crítico, latinoamericano y transformativo, analizando el impactos socio-culturales de proyectos globales sobre espacios locales y la capacidad de reacción de actores locales sobre proyectos globales.

Para la antropología el presente etnográfico, es el lugar y lo local, a partir de sus relaciones internas, con fronteras cerradas y vaciadas de la historia compleja. Sin embargo, otras perspectivas como Nash (1981, 1994, 2008), Shalins (1988), Augé (1994, 2007), Marcus (1986), Friedman $(2001,2014)$, se orientan a pensar «lo local» desde su interacción con «lo global», en el marco del sistema-mundo. Por otro lado, las visiones triunfalistas de la globalización (sobre todo las integradoras) se suman a la visión de la globalización como marea que arrastra disolviendo «lo local». La mirada abstracta y triunfalista de la globalización neutraliza la comprensión de las verdaderos impactos sociales, culturales, identitarios y en el territorio de lo global sobre lo local. Necesitamos una mirada crítica que permita captar esa complejidad tensa y dinámica, configurada por nuevos actores (bancos, corporaciones globales), arquitecturas institucionales, geografías de poder y redes transnacionales (Suárez, 2018). En este punto los trabajos de Saskia Sassen son sustanciales.

En mi visión, una antropología sistémica, anclada en las lógicas del sistema-mundo, el pensamiento crítico latinoamericano y la teoría critica transformativa, podría permitirnos una nueva aproximación de las relaciones locales y globales, examinando las complejas relaciones de fuerza, antagonismo entre actores y conflictos sociales y culturales que surgen en los espacios locales a partir de su articulación con lo global (Suárez, 2018). Esto se expresa como escala, ensamblaje institucional, redes de corporaciones transnacionales y Estados nacionales. Los estudios de caso demuestran que lo global supone una experiencia local, al mismo tiempo que existe un complejo entramado de relaciones de fuerza en ambas direcciones de lo global a lo local y de lo local a lo global, con mediaciones estatales y subnacionales.

La antropología sistémica surge ante la necesidad de repensar las implicancias sociales y culturales de la «Globalización», en los territorios locales. Al mismo tiempo que busco superar las visiones triunfalistas de la globalización (como integradora, mercado-céntrica), o la visión de la globalización como disolvente de las formas locales de vida. Busco consolidar una aproximación que supere dichas visiones limitadas de la realidad socio-cultural y permita develar el complejo sistema de fuerzas, actores e intereses, articuladas en distintas escalas. Por esa razón considero que la antropología sistémica apoyada en una etnográfica multisituada debe tomar como punto de partida lo global como experiencia concreta, situada en la densidad histórica, cargada de significados y sentidos, de relaciones asimétricas y fricción, también como experiencia de resistencia, lucha de poder y hegemonía. Necesitamos dar cuenta de esa compleja arquitectura global donde el crecimiento económico coexiste con las lógicas brutales de capitalismo expresadas en las expulsiones (Sassen, 2015) y el capitalismo de casino. La antropología sistémica nos ubica en lógica del sistema-mundo y es capaz de construir una voz desde los Andes, a fin de darle continuidad al pensamiento crítico latinoamericano (Mignolo, Lander, Quijano) compartiendo las aspiraciones de una teoría crítica transformativa.

Esta propuesta busca repensar el abordaje de las relaciones complejas entre lo global y lo local. Asumiendo que son interacciones dinámicas, tensas, cooperativas y conflictivas, a la luz de nuevas arquitecturas institucionales, geografías de poder, redes transnacionales (trabajo, capital, recursos) y actores que interactúan (Suárez, 2018). En nuestra visión, la globalización se hace palpable en lo local, al mismo tiempo que lo local puede ser visible a nivel global. En este trabajo defendemos la necesidad de una antropología sistémica de la mano de una etnografía multisituada (articulando escalas y registros), que 
permita abordar la complejidad global-local y sus repercusiones en la cultura, la identidad y el territorio, sin soslayar una mirada del sistema-mundo y la hegemonía de las corporaciones y las redes transnacionales. La antropología sistémica busca reivindicar el lugar de enunciación y pensar desde los andes, como un ejercicio anclado en el pensamiento crítico latinoamericano, sin descuidar las posibilidades de la teoría critica transformativa.

Desde la modesta tienda de antropólogo mi propuesta se encamina a problematizar las relaciones entre lo global-local y repensar la visión unívoca de arriba a bajo, o de abajo hacia arriba. Una antropología sistémica busca volver la mirada a una multiplicidad de problemas entre lo global-local desde la lógica de sistema-mundo, flujos complejos, dinámicas de crisis, retroalimentaciones, desplazamientos y reconfiguraciones. No es un sistema uniforme, ni estable, ni homogéneo y menos aun tendiente al equilibrio. Puede ser muy provechoso pensar la globalización como parte del complejo sistema-mundo, y como bien sugiere Sassen, encontraremos actores que se desplazan creando geografías de poder, creando territorios, debilitando formas de gobierno local, estimulando expulsiones de territorios, para favorecer la emergencia de nuevos territorios fértiles para la extracción de recursos o la financiarización de los alimentos básicos (Sassen, 2007), (Sassen, 2010), (Sassen, 2015). Desde hace ya un tiempo estamos evidenciando cómo la tecnocrática internacional cambia el rumbo a las políticas estatales. En estos tiempos ocurren una serie de fenómenos que están cambiando aceleradamente la dinámica del capitalismo neoliberal, por ejemplo, la digitalización de la economía, e-commerce, la emergencia de la economía colaborativa, la emergencia de la nano-tecnología que está cambiando el rumbo tanto de la biología como de la guerra.

La antropología sistémica reivindica la necesidad de repensar la interacción entre las diversas escalas: local, regional, nacional, transnacional y global. En ese sentido, si bien nos apoyamos en idea de sistema, no queremos caer en la abstracción extrema sino referirnos a dominios concretos de la experiencia. La propuesta que compartimos sugiere que los procesos y dinámicas del capital global sean analizados e interpretados desde la experiencia local (Suárez, 2018), a partir de sus efectos reales en los procesos vitales, significativos y relacionales de los sujetos y las colectividades en distintas escalas. Una mirada sistémica requiere pensar de manera dialéctica el sistema de fuerza y tensiones que atraviesan las distintas escalas de la vida social y por último toda explicación debe pasar por reconocer que existe una multi-causalidad y no una causalidad lineal, lo cual debe ser reintegrada en la mirada de proceso (Suárez, 2018). Una antropología sistémica va más allá de la falsa antinomia entre «modo de producción» $\mathrm{y}$ "modos de vida» (como se ha pensado muy tradicionalmente la cultura). $Y$ por ende no queremos seguir pensado que hay una desvinculación entre estructura o sistema y la experiencia de los sujetos, entre lo macro y lo micro. Aunque hay admitir que todo intento de teorización es también un intento de abstracción, la propuesta no debe desvincularse con los hechos sociales y las experiencias culturales concretas que están asociadas al sentido, al significado, al mundo de la vida, etc. Por esa razón, no queremos pensar en sistema como algo abstracto sino como un complejo de fuerzas expansivas de alcance global del capitalismo, sus lógicas brutales, sus actores y dinámicas de interacción con las distintas escalas (nacional, sub-nacional y local) hasta las experiencias locales.

La antropología sistémica de cierta forma se alimenta de las reflexiones epistemológicas de mayor alcance. En ese sentido, el pensamiento sistémico constituye una salida al pensamiento reduccionistamecanicista propio del siglo XVIII y XIX, un enfoque que busca ir más allá de la tradición aristotélica y mecanicista, donde la verdad se erige como universal y donde el trabajo científico es lineal, mono-causal (causalidad lineal), dicotómico, reduccionista y deductivo. Este enfoque sistémico busca situarse más allá de todo intento de reduccionismo. A su manera, se orienta a mostrar la interdependencia entre las estrategias, en este caso de los actores, y las estructuras, las mismas que son dinámicas y que obedecen a procesos de larga duración. Un pensamiento sistémico hace énfasis en la interacción de los elementos (aspecto sincrónico), los procesos de cambio (aspecto diacrónico), las conexiones probables y la condiciones que favorecen el mantenimiento del sistema o su cambio. Esto es posible si identificamos que existen límites del sistema, conexiones y dinámicas de interretroalimentación, así como puntos de influencia permitiendo que el sistema opere y se transforme 
(Pirri \& Di Bacco, 2015). Desde una perspectiva sistémica, Jacques Lapointe (1993) nos recuerda que el planteamiento de Jean-Louis Le Moigne sobre lo sistémico supone reconocer el papel del investigador, es quien define cada objeto que estudia a partir de sus intenciones explícitas o implícitas; que cada objeto, que es conocido por nuestro proceso cognitivo, es parte inmersa y activa dentro de un todo más grande. Por esa razón, el sistema como un todo es mayor que sus partes, a esto se le llama globalismo. El enfoque sistémico trata de no usar el concepto total, no habla de un sistema total, sino de un sistema global, pues asumen que es un conjunto que no se puede reducir a la suma de sus elementos. A esto se agrega que la estructura del sistema refleja los diferentes puntos de vista, proyectos y lógicas de los actores que son parte del proceso y que poseen una naturaleza altamente dinámica. Otra idea importante, es interpretar el objeto no en sí mismo sino por su comportamiento y recursos que moviliza. El sistema nunca está del todo acabado y pueden agregarse más elementos a la luz de nuevos resultados; por esa razón, la perspectiva sistémica supone que existe un intercambio de recursos u otros elementos que permite al sistema mantenerse y operar. Por otro lado, el enfoque sistémico enfatiza las transformaciones, los cambios, las permutaciones, por eso es necesario ver las cosas de manera diacrónica y multicausal. Para la lógica sistémica, estos cambios tienen relación con elementos nuevos que ingresan al sistema, al entorno, por ende, se produce una suerte de dinámica de entrada y salida. De ahí que el enfoque sistémico esté muy relacionado con el concepto de flujos de energía, de información, de códigos, de sentidos.

Otro aspecto importante es la interacción entre cada uno de los elementos del sistema, a eso se le conoce como redundancias interactivas. Según Dominique Bériot (2006), estos pueden ser pensados como intercambios y comportamientos, y aunque a veces se dan de manera espontánea e inconsciente en realidad están estructurados y se desarrollan según reglas subyacentes (Bériot, 2014). El enfoque sistémico toma en consideración la existencia de múltiples circuitos internos de retroalimentación: bucles de retroalimentación negativos, positivos y pos-antagónicos. En la visión del enfoque sistémico se trabajan estos bucles en red, a fin de que su interacción logre cierta estabilidad y adaptarse a procesos de cambio, y es ahí cuando el sistema se percibe en movimiento. Los bucles de retroalimentación (boucles de rétroaction) facilitan la dinámica del cambio y la posibilidad de reinyección, esto propicia y amplificar la transformación en curso. Así mismo, existen efectos acumulativos como «bola de nieve» que facilita la expansión (o a veces el bloqueo de la actividad). Por otro lado, existen bucles negativos o estabilizadores que favorecen el equilibrio y la estabilidad. Aquí, la retroalimentación actúa en la dirección opuesta a la diferencia de equilibrio (punto de ajuste) de la variable de salida. No hay duda que la interacción de ambos bucles juntos puede ser contradictorio y hasta paradójico para una mente lineal y binaria. Por eso se dice que existe cierto pos-antagonismo que le permite al sistema salir de su equilibrio.

La antropología sistémica debe tomar consideración ese lado perverso y contradictorio de la globalización, sin descuidar la comprensión de múltiples experiencias locales. En último término reclamamos la importancia de analizar las implicancias de las fuerzas globales sobre las prácticas y los procesos vitales de sentido y significado. En esa dirección, el trabajo de Saskia Sassen es de notable importancia. Su obra nos permite ir más allá de la versión hegemónica de la globalización, concebida usualmente como algo abstracto y omnipresente de fuerzas. Sassen desarrolla una sociología rigurosa y no se fía de ningún tipo de moda académica, ni teórica. Desde mi perspectiva, su contribución reside en su ejemplo de inconformismo, su rigurosidad y anti-establisment. Su táctica analítica le permite moverse entre los bordes sistémicos y superar las "categorías maestras", mostrando sus limitaciones y perversidades.

Una antropología sistémica se apoya en la interdisciplinariedad como principio de acción epistémica. Nuestra propuesta se inclina por la renovación de las ciencias sociales, con un aire fresco, crítico y heterodoxo que permita mirar atrás para avanzar hacia delante retomando la contribución de maestros de la sospecha como Fernand Braudel y construir un diálogo fructífero con las contribuciones de Giovanni Arrighi, Samir Amin y otras miradas heterodoxas por ejemplo Yanis Varoufakis (Suárez, 2018).

La antropología sistémica le interesa el complejo nivel de estructuración y dinámica sistémica del capitalismo global y sus fuerzas, asumiendo que esto constituye una pista en doble sentido. Esta propuesta 
busca apoyarse en un ejercicio de etnografía multisituada, en diversos círculos antropológicos ya se viene hablando de esta propuesta que se enmarca sobre todo en el estudio de procesos globales (Freidberg, 2001) y muy especialmente en los estudios sobre migraciones, diásporas y redes transnacionales (Berg \& Paerregaard, 2005), (Berg, 2017). Uno de los promotores de este ejercicio es Ulf Hannerz y George Marcus, quien sugirió una suerte de etnografía en el sistema-mundo (Marcus, 1995), (Hannerz, 2003), (Bélanger-Vincent, 2011), (Marcus, 2012), (Falzon, 2016). Desde mi perspectiva, una propuesta de etnografía multisituada debe articular dos planos, por un lado, el plano de las escalas territoriales como lo local, subnacional, nacional y global ubicando la interacción entre actores y territorios; y por otro lado, el plano, donde ubicamos las nuevas articulaciones globales. De este modo se puede explorar el proceso por el cual se construyen políticas, discursos y prácticas de orden global, también podemos desplazarnos por las prácticas y discursos tecno-burocrático. Así mismo es pertinente que tomemos en consideración el rol que juegan los medios masivos de control de la opinión publica y la información y decantar hasta el plano de las voces locales, sus demandas, sus experiencias significativas, los desplazamientos y reestructuraciones a un nivel más profundo. En ese sentido, la etnografía multisituada no solo contempla una variedad de actores y procesos, además, puede articularse con una diversidad de registros.

La propuesta de la antropología sistémica se nutre del diálogo entre las ciencias, como un proceso de articulación epistemológica que permite obtener lo mejor de cada una de las miradas y estimule el surgimiento de un paradigma más avanzado para la comprensión de sistemas complejos. Desde mi punto de vista, no hay otra posibilidad para el avance de las ciencias sociales y en parte, retomando el argumento de Wallerstein, debemos sacudirnos un poco de esta mirada compartimentada que se ha creado en las ciencias y que de cierta forma impacta sobre la manera cómo se ha organizado la academia y las ciencias sociales, para poder reorganizarnos a partir de problemas comunes, apoyándonos en un pensamiento convergente.

Una antropología sistémica debe reconocer la voz y el lugar de enunciación, al tiempo que se conecta con toda una tradición de pensamiento crítico lati- noamericano. De cierta manera, refuerza un sentido de comunidad latinoamericana y desde el sur global. Aquí es importante conectar con la idea de heterogeneidad histórico estructural y revisitarla en un contexto de bucles de retroalimentación del sistema capitalista global. Reconocer que vivimos mundos heterogéneos es algo que no solo se ha reconocido en Latinoamérica también en la India y otros países poscoloniales. De ahí la importancia de leer esta complejidad sistémica y estructural desde la decolonialidad como lo han sugerido Quijano, Mignolo, Dussel, entre otros.

Una antropología sistémica también se plantea la pregunta ¿para qué? ¿qué sentido tiene repensar y trabajar una arquitectura conceptual? Desde mi posición, esta perspectiva puede contribuir a imaginar otros escenarios posibles de cambio y transformación social desde abajo. Pensar de manera sistémica y compleja, busca explorar qué posibilidades tenemos para hacer cambios sociales considerando la emergencia de otras alternativas sociales, políticas y económicas desde el sur global. La propuesta de antropología sistémica es una respuesta crítica al conformismo y escapismo intelectual. Aunque nuestra idea no es quedarnos en el plano de la denuncia, es necesario ir a la propuesta, ¿cómo hacerlo? A partir de principio de transdisciplinariedad, de diálogo entre saberes, y trabajo colaborativo (co-laboratorios) como principio y posibilidad de imaginar otros mundos posibles.

Finalmente, una antropología sistémica no puede seguir pensando lo social evitando la ebullición de nuevas tendencias en la ciencia, la tecnología, la ingeniería y las sinergias con el sistema capitalista global. Así, la digitalización de la sociedad, el desarrollo de la nano-tecnología, la minería de datos, la búsqueda de exoplanetas, la inteligencia artificial (IA), etc., constituyen problemas de investigación que merecen ser analizados a partir de su sinergia con las fuerzas globales del capitalismo, sus fuerzas expansivas, sus actores y sus implicancias para procesos vitales de colectividades e individuos en sus diversos y amplios campos. Solo para citar un ejemplo, la economía colaborativa viene usando la inteligencia artificial y la digitalización como una estrategia para estimular dinámicas de acumulación a escala global, estimulando la precarización de miles de trabajadores que se multiinsertan en esta era del trabajo globalizado. Una antropología sistémica debe ser capaz de identificar los riesgos del 
porvenir y las potencialidades para la transformación social, en medio de los avances tecnológicos y científicos, de modelos como la economía colaborativa y las mediaciones tecnológicas propias de una sociedad que se digitaliza. Desde una perspectiva crítica, nuestra perspectiva puede contribuir con un giro de timón desde el sur global.

\section{Bibliografía}

Basu, P. K. (2007). Political economy of land grab. Economic and Political Weekly, 1281-1287.

Bateson, G. (1979). Mind and nature: A necessary unity. New York: Bantam Books.

Bélanger-Vincent, A. (2011). Multisited Ethnography. Theory, Praxis, and Locality in Contemporary Research. Anthropologica, 53(1), 181.

Berg, Ulla (2017). Mobile selves: race, migration, and belonging in Peru and the US. NYU Press.

Berg, Ulla \& Paerregaard, K. (2005). El 5 to suyo. Instituto de Estudios Peruanos.

BÉriot, D. (2014). Manager par l'approche systémique: Préface de Michel Crozier. Editions Eyrolles.

De Rosnay, J. (2014). Le macroscope. Vers une vision globale. Le seuil.

Donnadieu, G. (2004). Systémique et science des systèmes: quelques repères historiques. Association Française de Systémique (AFSCET).

Donnadieu, G. e. (2003). «L'Approche systémique: de quoi s' agit-il.» Synthèse des: AFSCET, Groupe.

Donnadieu, G., \& Karsky, M. (2002). La systémique, penser et agir dans la complexité. Ed. Liaisons.

FALzON, M.-A. (2016). Theory, praxis and locality in contemporary research. Routledge.

Feenberg, A., \& Callon, M. (2010). Between reason and experience: Essays in technology and modernity. MIT Press.

FLood, R. L. (2010). The relationship of 'systems thinking' to action research. Systemic Practice and Action Research, 23(4), 269-284.

Freidberg, S. (2001). On the trail of the global green bean: methodological considerations in multi-site ethnography. Global Networks, 1(4), 353-368.

Hannerz, U. (2003). Being there... and there... and there! Reflections on multi-site ethnography. Ethnography, 4(2), 201-216.

Harvey, D. (2013 de Diciembre de 2016). Lefteast. David Harvey: "There is no way you can change the world without changing your ideas!», págs. http://www.criticatac.ro/lefteast/david-harvey-interview-2016/.

KaKu, M. (2015). The future of the mind: The scientific quest to understand, enhance, and empower the mind. Anchor Books.

KaKu, M. (2016). Hyperspace: A scientific odyssey through parallel universes, time warps, and the tenth dimension. Oxford University Press.

Klein, N. (2017). Decir no. No basta. Barcelona: Paidós.

Krugman, P. (11 de Diciembre de 2011). Somos el 99,9\%. El País.

Latour, B. (2005). Reassembling the social: An introduction to actor-network-theory. Oxford University Press.

Marcus, G. (1995). Ethnography in/of the world system: The emergence of multi-sited ethnography. Annual review of anthropology, 24(1), 95-117.

Marcus, G. (2012). Multi-sited Ethnography. En S. Coleman, \& P. Von Hellermann, Multi-Sited Ethnography: Problems and Possibilities in the Translocation of Research Methods.

Merton, R. (1968). Social theory and social structure. Simon and Schuster.

NAPOLEONI, L. (2008). Economía canalla: la nueva realidad del capitalismo. Grupo Planeta (GBS).

OXFAM. (2016). Una economía al servicio del $1 \%$. Oxfam-Intermon.

PALAN, R. (2006). The offshore world: sovereign markets, virtual places, and nomad millionaires. Cornell University Press.

Parsons, T. (1991). The Social System. With a New Preface by Bryan S.Turner. London: Routledge \& Kegan Paul Ltd.

Piketty, T. (2014). El capital en el siglo XXI. México: Fondo de cultura económica.

Pilkington, E., \& Smith, D. (6 de noviembre de 2017). Bernie Sanders warns of 'international oligarchy' after Paradise Papers leak. The Guardian, págs. https://www. theguardian.com/news/2017/nov/06/bernie-sandersparadise-papers-leak-international-oligarchy.

Pirotton, G. (2005). Introduction à la systémique. Boîte à outils conceptuels pour aider la réflexion, la formation et l'action des écologistes et de tous ceux qui sont tentés par une compréhension globale de la complexité. Etopia, http://www.etopia.be/IMG/pdf/18.systemique.pdf.

Pirri, P., \& Di BAcco, M. (2015). Il pensiero sistemico: un approccio alla complessità.

Richardson, D. (11 de junio de 2017). 5 Revelations About Global Oligarchy From the Paradise Papers. 
observer.com, págs. http://observer.com/2017/11/ revelations-about-the-global-oligarchy-from-the-paradise-papers/.

Ritzer, G. (2003). Teoría sociológica moderna. Madrid: Mc Hill.

SaHlins, M. (1994). Cosmologies of capitalism: The transpacific sector of "The World System». Culture/power/history: A reader in contemporary social theory, 412-455

SAsSEN, S. (2007). Una sociología de la globalización. Buenos Aires: Editorial Katz.

Sassen, S. (2010). Territorio, autoridad y derechos: de los ensamblajes medievales a los ensamblajes globales. Katz editores.

SASSEN, S. (2015). Expulsiones: brutalidad y complejidad en la economía global. Buenos Aires: Katz editores.

Sloterdijk, P. (2014). Esferas III: Espumas. Esferología plural. Siruela.
SuÁRez Rojas, Luis Alberto (2018). Antropología Sistémica. Aproximaciones criticas de lo global/local con una perspectiva poscolonial desde los Andes. Lima: Tesis Doctoral. Universidad Nacional Mayor de San Marcos.

Tilly, C. (2000). La desigualdad persistente. Buenos Aires: Manantial.

Turchany, G. (2008). La théorie des systèmes et systémiques: Vue d'ensemble et définitions. Rpoceedings of Conférence Internationale de Bordeaux. Vol. 27.

URRY, J. (2017). Offshore. La deslocalización de la riqueza. Madrid: Capitán Swing.

VISSER, M. (2003). Gregory Bateson on deutero-learning and double bind: A brief conceptual history. Journal of the History of the Behavioral Sciences, 39(3), 269-278.

Volscho, T., \& Nathan, K. (2012). The rise of the superrich: Power resources, taxes, financial markets, and the dynamics of the top 1 percent, 1949 to 2008. American Sociological Review, 77(5), 679-699. 